ఠ

\title{
Role of human beta defensin 3 during type I interferon mediated antiviral response against vesicular stomatitis virus
}

This article was published in the following Dove Press journal:

International Journal of Interferon, Cytokine and Mediator Research

26 February 2010

Number of times this article has been viewed

\author{
Mausumi Basu² \\ Srikanth Kota' \\ Amiya K Banerjee ${ }^{2}$ \\ Santanu Bose' \\ 'Department of Microbiology and \\ Immunology, The University of Texas \\ Health Science Center at San Antonio, \\ San Antonio, TX, USA; ${ }^{2}$ Department \\ of Molecular Genetics, Section of \\ Virology, Lerner Research Institute, \\ Cleveland Clinic, Cleveland, OH, USA
}

\begin{abstract}
Type I interferons (IFN), IFN- $\alpha / \beta$ constitute a critical component of innate immunity against viruses. IFN- $\alpha / \beta$ activates JAK/STAT pathway resulting in expression of various IFNstimulated antiviral proteins. While studying IFN- $\alpha / \beta$ signaling during virus infection, we identified human beta defensin-3 (HBD3) as an antiviral factor induced during IFN- $\alpha$-mediated innate antiviral response in human lung epithelial cells. We showed that HBD3 is induced by IFN- $\alpha$ and purified HBD3 significantly inhibited vesicular stomatitis virus (VSV) infection. Further studies revealed that HBD3 confers its antiviral activity by blocking VSV cellular entry. An essential role of HBD3 during innate antiviral response was evident from loss of antiviral activity of IFN- $\alpha$ following HBD3 silencing by siRNA. Thus, we have identified HBD3 as an IFN-inducible antiviral factor that inhibits infection by blocking viral cellular entry.

Keywords: innate immunity, type I interferon, human beta defensin, virus infection
\end{abstract}

\section{Introduction}

Interferons (IFNs) constitute an important molecule of innate immune response induced by host to control virus infection. ${ }^{1,2}$ Type I IFN (IFN- $\left.\alpha / \beta\right)$ and type-II IFN (IFN- $\left.\gamma\right)$ are potent antiviral cytokines that are produced from both nonimmune (eg, epithelial cells) and immune cells. IFN- $\alpha / \beta$ mediates its antiviral activity by binding to IFN receptor on the cell surface, which leads to activation of receptor-associated Janus kinases (JAK)/signal transducers and activators of transcription (STAT) pathway. Activation of JAK/STAT pathway results in expression of IFN-stimulated genes (ISGs). Several ISGs are antiviral factors, including oligoadenylate 2-5A synthetase (OAS)/RNase L, ${ }^{3}$ dsRNA activated protein kinase (PKR), ${ }^{4}$ and the Mx proteins (Mx1 in mice and MxA in humans). ${ }^{5}$ These IFN-induced antiviral proteins restricts a wide spectrum of viruses including single-stranded nonsegmented negative strand RNA (NNS) viruses. ${ }^{6-9}$

Enveloped NNS viruses represents viruses such as vesicular stomatitis virus (VSV), human parainfluenza virus type 3 (HPIV-3) and human respiratory syncytial virus (RSV). It is known that upon IFN- $\alpha / \beta$ treatment, three well-established ISGs (OAS/ RNaseL, PKR, Mx) are capable of restricting NNS virus infection. However, several studies have provided evidence for the existence of alternative IFN-induced factors that are required for the antiviral activity of IFN- $\alpha / \beta$. It was reported that IFN confers its antiviral activity against HPIV-3 independent of PKR, Mx and RNaseL. ${ }^{10}$ In addition, studies with PKR, Mx and RNaseL triple knockout mouse embryonic fibroblasts demonstrated that IFN- $\alpha / \beta$ retains its antiviral activity against VSV in the absence of PKR, Mx and RNAseL. ${ }^{11}$ Similarly, the ability of IFN to restrict VSV replication in cells expressing PKR, RNase L, and Mx proteins has been reported previously. ${ }^{12}$
Correspondence: Santanu Bose Department of Microbiology and Immunology, The University of Texas Health Science Center at San Antonio 7703 Floyd Curl Drive, MC-7758, San Antonio, TX 78229, USA

$\mathrm{Tel}+\mathrm{I} 2105671019$

Email bose@uthscsa.edu 
Moreover, we have previously shown that type I IFN is capable of inducing antiviral factors like phospholipid scramblase- $1^{13}$ and soluble secreted factors. ${ }^{14}$ These studies strongly suggest the existence of additional IFN- $\alpha / \beta$ induced antiviral factors that are operational during innate immune response against NNS viruses.

In an effort to identify type-I interferon induced antiviral factors; we investigated the antiviral mechanism of IFN- $\alpha$ in human lung epithelial A549 cells infected with VSV, a virus highly sensitive to the antiviral action of IFN- $\alpha / \beta^{15}$ Our studies revealed that human beta defensin-3 (HBD3) induced by IFN- $\alpha$ possess significant antiviral activity. Defensin family of polypeptides comprising of alpha, beta and theta defensins, possess a wide range of antipathogenic activity against bacteria and viruses. ${ }^{16-18}$ Among them human beta-defensins (HBDs) are small epithelial cell-specific peptides that are secreted by epithelial cells to restrict infection during innate immune response. ${ }^{17}$ Recently, antiviral activity of HBDs against human immunodeficiency virus-1 (HIV-1) and influenza virus has been reported. ${ }^{19,20} \mathrm{We}$ have also recently identified HBD2 as a nuclear factor- $\kappa \mathrm{B}(\mathrm{NF}-\kappa \mathrm{B})$-dependent antiviral factor that inhibited RSV infection by virtue of blocking RSV cellular entry. ${ }^{21}$ In this communication we demonstrate that HBD3 is an IFN- $\alpha$ inducible protein that restricts VSV infection by inhibiting cellular entry of the virus into A549 cells.

\section{Materials and methods}

\section{Virus, cells, and other reagents}

VSV (Indiana serotype, Mudd-Summers strain) was propagated in BHK-21 cells. ${ }^{14,22}$ Human lung epithelial A549 cells were maintained in DMEM (GIBCO-BRL, Gaithersburg, MD, USA) supplemented with $10 \%$ fetal bovine serum (FBS), penicillin, streptomycin, and glutamine. The viral titer was monitored by plaque assay analysis with CV-1 cells as described earlier. ${ }^{14,22}$ IFN and TNF were purchased from R\&D Systems (Minneapolis, MN, USA).

\section{Western blot analysis}

Following 7.5\% SDS-PAGE and transfer of proteins to the nitrocellulose membrane, the membrane was blocked with blocking buffer. Anti-VSV P protein antibody (polyclonal) was added to the blot in the presence of the blocking buffer and incubated overnight at $4{ }^{\circ} \mathrm{C}$. The washed blots were incubated with anti-rabbit-horseradish peroxidase (HRP) and processed for enhanced chemiluminescence according to the manufacturer's protocol (Amersham-Pharmacia, Piscataway, NJ, USA).

\section{VSV infection following HBD treatment}

To study the antiviral function of HBD3, A549 cells were pre-treated with either HBD1 (control) or HBD3 (12 $\mu \mathrm{g} / \mathrm{ml})$ in DMEM (-FCS) or $10 \mathrm{mM}$ phosphate buffer for three hours at $37^{\circ} \mathrm{C}$. Following incubation, VSV (0.5 MOI) was added to the washed cells and the adsorption was continued for 1.5 hours at $37^{\circ} \mathrm{C}$ either in the presence or absence of HBDs. The cells were then washed to remove unbound viruses and the infection was continued for additional $36 \mathrm{~h}$ (in the absence of HBDs) in complete (DMEM + 10\% FCS) medium. Thirty-six hours post-infection, the cell lysates were used for Western blot analysis with anti-VSV P protein antibody to monitor the intracellular VSV P protein levels. In addition, the culture supernatants were collected to measure virus yield by plaque assay analysis on CV-1 cells. The homogeneous preparations of purified HBDs utilized in these studies were either obtained as a gift from Dr. Miguel E. Quinones-Mateu (Cleveland Clinic Foundation, Cleveland OH, USA), who used them to study anti-HIV-1 activity ${ }^{19}$ or purchased from PeproTech (Rocky Hill, NJ, USA).

\section{Immunofluorescent staining}

A549 cells (grown on coverslips) were either untreated or incubated with IFN- $\alpha$ (1000 units/mL) for eight hours at $37{ }^{\circ} \mathrm{C}$. The cells were then washed with $1 \times$ phosphatebuffered saline (PBS) and fixed with 3.7\% formaldehyde in PBS at room temperature and permeabilized with $0.5 \%$ Triton X-100. The cells were stained with anti-HBD3 antibody (Orbingen, San Diego, CA, USA) and anti-rabbit IgG conjugated to FITC. The coverslips were mounted on slides with Vectashield and visualized in a Leica CISM confocal laser-scanning microscope.

\section{VSV G pseudotyped lentivirus expressing eGFP (lenti-VSV)}

To study the cellular entry efficiency of VSV in HBD3-treated cells, we have constructed VSV envelope protein (G protein) pseudotyped lentivirus expressing the enhanced GFP (eGFP) gene (lenti-VSV). ${ }^{14}$ The construction lenti-VSV was achieved by cloning eGFP gene in the BamH1 and EcoRV sites of $6.9 \mathrm{~kb}$ lentiviral vector under the control of CMV promoter (plenti/V5-Dest, Invitrogen) and transfecting this vector in 293 cells along with HIV-gag-pol, HIV-Rev and VSV G plasmids. Seventy-two hours post-infection, the infectious eGFP-expressing pseudotyped viruses possessing 
VSV G on its envelope were purified from the supernatant. The transduction efficiency of these viruses, representing cellular entry via VSV G protein was tested in HBD-treated A549 cells. For these studies, A549 cells were either untreated or incubated with HBD1 and HBD3 $(12 \mu \mathrm{g} / \mathrm{ml})$ for eight hours. The cells were then washed and lenti-VSV (15 MOI) was added in the absence of HBDs (in the presence of complete medium). Forty hours post-transduction, eGFP expression was monitored by fluorescence microscopy.

\section{Real-time quantitative RT-PCR (qPCR) assay to quantify HBD mRNA}

RNA from A549 cells incubated with IFN- $\alpha(1000 \mathrm{U} / \mathrm{mL})$, IFN- $\gamma(1000 \mathrm{U} / \mathrm{mL})$ and tumor necrosis factor- $\alpha$ (TNF- $\alpha 20$ $\mathrm{ng} / \mathrm{mL}$ ) was extracted using TRIzol (Invitrogen Life Technologies, Carlsbad, CA, USA). RNA content in each preparation was normalized using human keratin 5 RNA. Intron spanning primers and PCR conditions used for qPCR has been described previously. ${ }^{23,24}$ RNA generated by transcribing HBD1 and HBD3 plasmids (by using the RiboProbe in vitro transcription system from Promega (Madison, WI, USA) was used to generate standard curves. Amplifications and data acquisition/ analysis were performed by using the Smart Cycler System software (version 1.2d; Cepheid, Sunnyvale, CA, USA).

\section{Treatment of cells with siRNAs}

siRNA against HBD3 and HBD1 were designed based on the siRNA designing program provided on-line by Qiagen (Valencia, CA, USA). During these experiments, scrambled siRNA (control) served as a negative control. The HPLC purified siRNAs (control and HBD siRNAs were purchased from Qiagen) were transfected using the siRNA transfection kit (Qiagen) according to the manufacturer's protocol. At 36-hours post-transfection, cells were treated with IFN- $\alpha$ (for eight hours) and HBD expression was monitored by qPCR. For the infection studies, siRNA transfected cells were treated with IFN- $\alpha$ for $8 \mathrm{~h}$, followed by VSV infection (in the presence of IFN- $\alpha$ ) for 36 hours. Viral titer was monitored by plaque assay. Previously we have utilized scrambled control and HBD2-specific siRNAs (purchased from Qiagen) to silence genes in A549 cells. ${ }^{21}$

\section{Results}

\section{Induction of HBD3 mRNA and protein by IFN- $\alpha$}

The rationale for focusing on $\mathrm{HBD} 3$ as a putative IFN- inducible antiviral protein in epithelial cells resulted from several known properties of HBD3. HBD3 is an inducible protein harboring STAT1-binding sequences in its gene. ${ }^{25}$ Since STAT1 is activated by IFN- $\alpha / \beta$, we speculated that HBD3 may be induced following IFN- $\alpha$ treatment. Moreover, HBDs are expressed exclusively in respiratory epithelial cells, ${ }^{17,26-28}$ the cells (human lung epithelial A549 cells) utilized in our studies and it has been detected in various body fluids (respiratory secretions, saliva, blood). ${ }^{29,30}$

In order to examine induction of HBD3 mRNA by IFN, A549 cells were treated with various cytokines for eight hours and the cells were processed for qPCR analysis to detect HBD3 mRNA. While induction of HBD3 mRNA was not noted in IFN- $\gamma$ - and TNF- $\alpha$-treated cells, significant levels of HBD3 mRNA was observed in IFN- $\alpha$-treated cells (Figure 1A). In contrast to HBD3, the cytokines failed to induce HBD1 mRNA.

Since, IFN- $\alpha$ stimulated HBD3 mRNA, we next investigated whether such activation leads to HBD3 protein synthesis. For these studies, untreated and IFN- $\alpha$-treated (eight hours) A549 cells were used for immunofluorescence analysis using HBD3 specific primary antibody and anti-rabbit FITC labeled secondary antibody. Confocal microscopic analysis of these cells revealed significant increase in HBD3 protein levels in IFN- $\alpha$ treated cells, compared to untreated cells (Figure 1B). Lack of HBD3 protein induction was also noted in IFN- $\gamma$ and TNF- $\alpha$-treated cells (data not shown). Incubation of cells with IFN- $\alpha$ for only two hours lead to HBD3 induction, while shorter and/or prolonged exposure of cells to IFN- $\gamma$ and TNF- $\alpha$ did not result in HBD3 activation (data not shown). The results from these studies indicated that HBD3 is an IFN- $\alpha$ inducible protein. Since HBDs possess antiviral function against viruses like HIV-1, influenza and $\mathrm{RSV},{ }^{19-21}$ we next examined whether such function is also manifested against VSV.

\section{Pre-treatment of A549 cells with HBD3 results in the inhibition of VSV infection}

In order to examine whether defensins, especially HBD3 possess antiviral property against NNS viruses, we investigated the ability of purified HBDs to inhibit VSV infection. A549 cells incubated with HBD1 and HBD3 (10 or $20 \mu \mathrm{g} / \mathrm{ml}$ ) for three hours at $37^{\circ} \mathrm{C}$ were infected with VSV. Following virus adsorption for one hour in the presence of HBDs, fresh medium was added to washed cells in the absence of HBDs. At 36 hours post-infection, virus titer and infection efficiency was assayed by plaque assay 
A

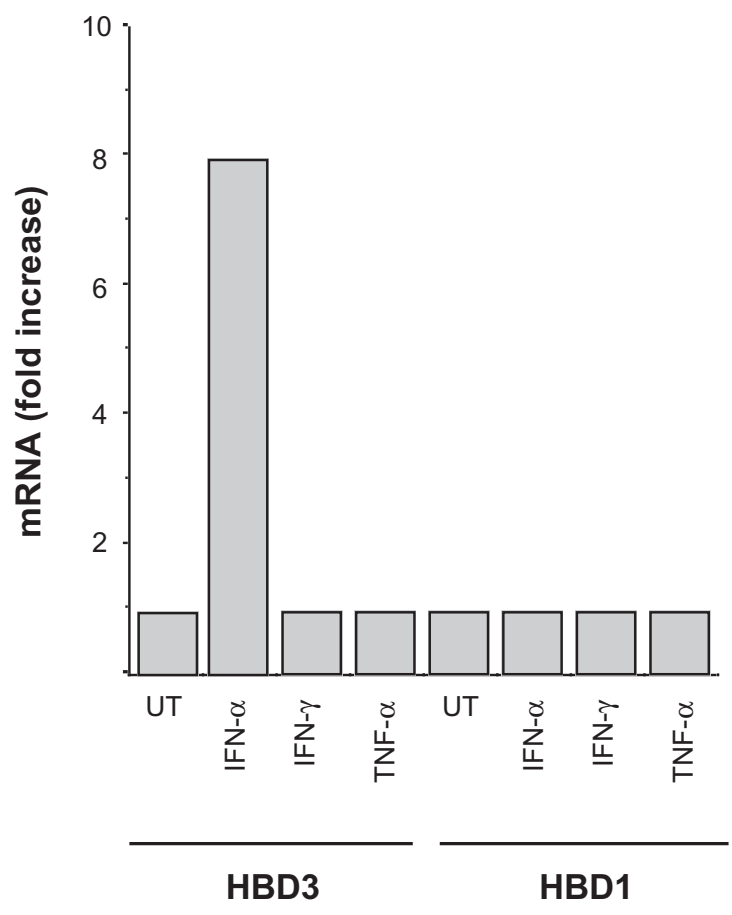

B

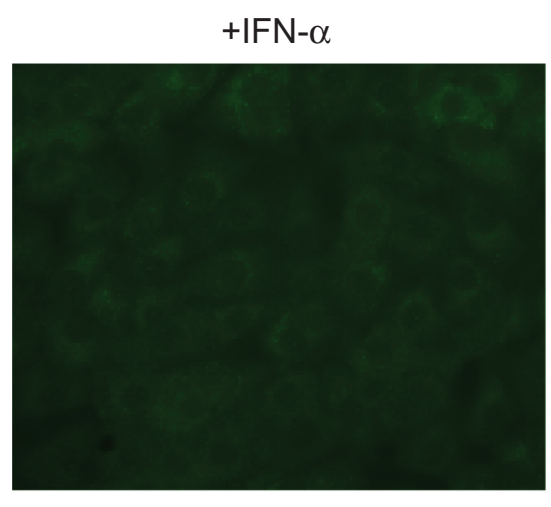

-HBD3 Ab

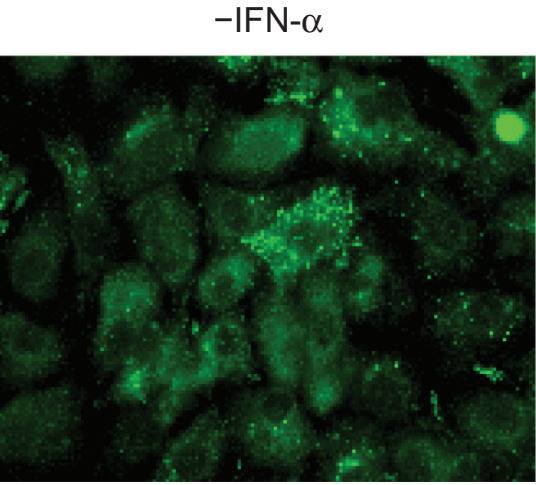

$+\mathrm{HBD} 3 \mathrm{Ab}$

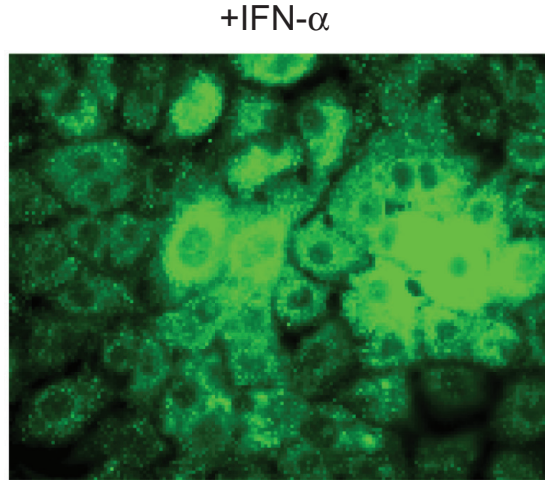

$+\mathrm{HBD} 3 \mathrm{Ab}$

Figure I Induction of HBD3 by type-I IFN. A) qPCR analysis of HBD3 and HBDI expression in untreated (UT) and TNF- $\alpha$, IFN- $\alpha$, IFN- $\gamma$-treated A549 cells. For the qPCR, the transcript levels were normalized to human keratin 5 and the results are representative of three independent experiments with similar values. B) Confocal immunofluorescence analysis of HBD3 expression in untreated (-IFN- $\alpha$ ) and IFN- $\alpha$-treated (+IFN- $\alpha$ ) A549 cells. HBD3 Ab; cells not incubated with antiHBD3 antibody (primary antibody), but processed with only secondary antibody (anti-rabbit lgG conjugated to FITC).

and Western blot (with anti-VSV P antibody) analysis. As shown in Figure 2A, plaque assay of medium supernatants obtained from HBD3-treated cells revealed significant (by 100-fold) decline in infectivity. In contrast such inhibition was not observed in HBD1-treated cells. Concomitantly, Western blot analysis (Figure 2B) of cell lysates with VSV $\mathrm{P}$ antibody demonstrated complete abolishment (since the virus titer was decreased by 100 -fold, corresponding to $99 \%$ inhibition in infectivity) of intracellular VSV P protein following treatment of cells with HBD3 $(20 \mu \mathrm{g} / \mathrm{mL})$. These results suggested that $\mathrm{HBD} 3$ possess potent antiviral activity against VSV.

\section{Incubation of VSV with HBD3 results in loss of virus infectivity}

Apart from the ability of defensins to restrict virus entry presumably by coating the cell surface following incubation of cells with defensins, it was also shown that incubation 
A

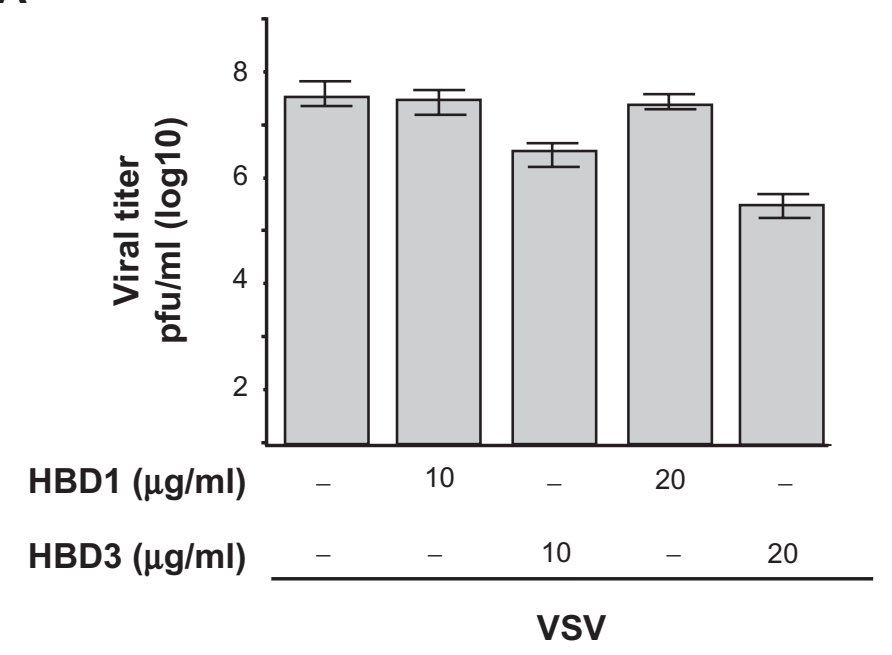

C

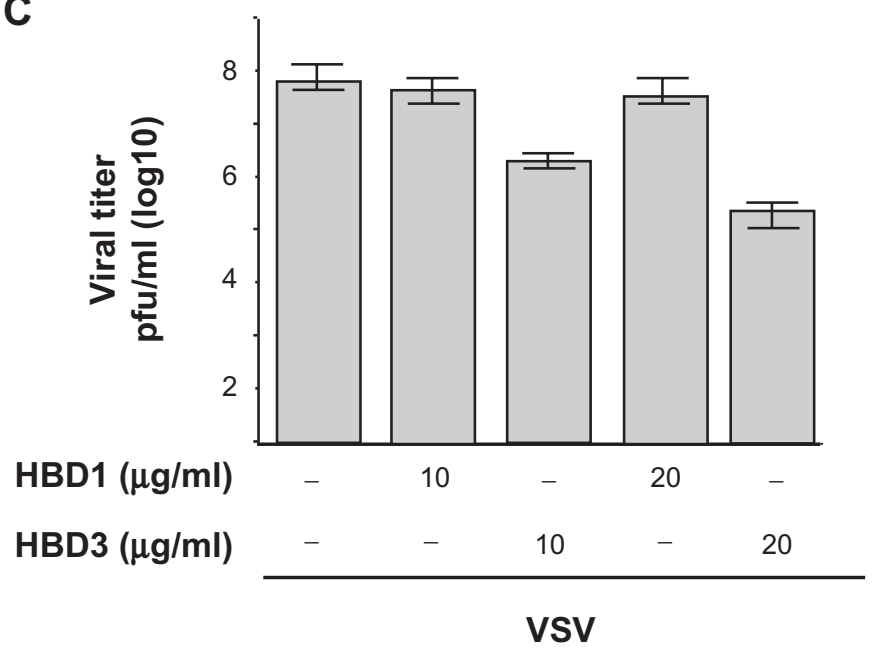

B

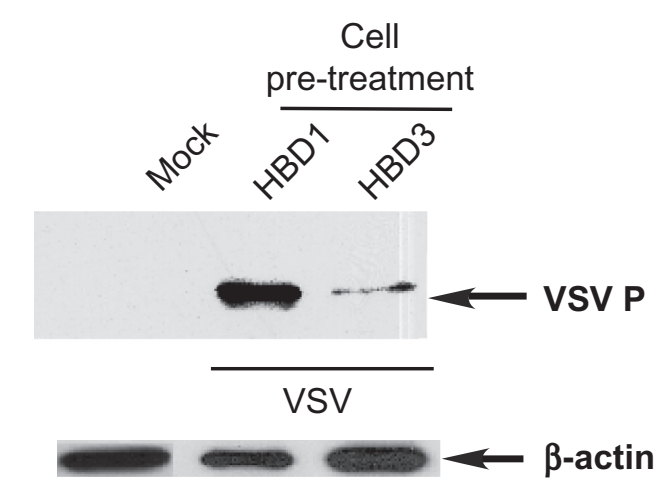

D

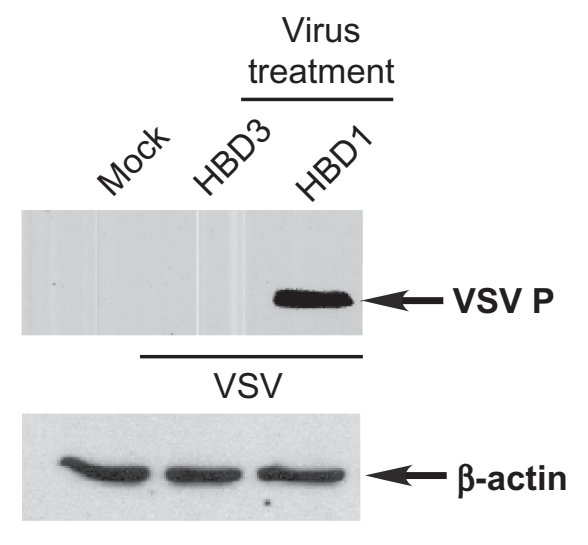

Figure 2 Antiviral activity of HBD3. A) VSV titer in A549 cells treated with indicated concentrations of HBDI and HBD3 proteins. The plaque assay values represent the mean \pm standard deviation for three determinations. B) Western blot analysis of VSV P protein expression in infected A549 cells treated with either HBD I or HBD3. C) VSV titer in A549 cells infected with virus that was pre-incubated with indicated concentrations of HBDI and HBD3 proteins prior to infection. The plaque assay values represent the mean \pm standard deviation for three determinations. D) Western blot analysis of VSV P protein expression in A549 cells infected with VSV that was pre-incubated with either HBDI or HBD3 prior to infection.

of virion particles with HBDs permeabilizes (perforates/ disrupts) the viral envelope lipid bilayer, thus rendering noninfectious virion particle that fails to enter the cells. ${ }^{12,20,21}$ To explore these possibilities, purified VSV particles $(0.5$ MOI) incubated with HBD1 and HBD3 (10 or $20 \mu \mathrm{g} / \mathrm{mL}$ ) for two hours at $37^{\circ} \mathrm{C}$ were added to A549 cells. Following $1 \mathrm{~h}$ adsorption, fresh medium was added to washed cells in the absence of HBDs. As shown in Figure 2C, plaque assay analysis of medium supernatants obtained from cells infected (36 hours postinfection) with untreated, HBD1or HBD3-treated VSV revealed significant (by 100-fold) decline in infectivity following HBD3 treatment of virion particles. In contrast, such inhibition was not observed following incubation of VSV with HBD1. Similar to the plaque assay result, Western blot analysis (Figure 2D) also demonstrated the loss of infectivity of HBD3-treated VSV as evident from the absence of VSV P protein in cells infected with these virus particles. In contrast to the potent 
antiviral action noticed following treatment of cells/viruses with HBD3, it failed to inhibit virus replication when it was added after (4-8 hours postinfection) virus infection (in the absence of HBD3 treatment prior to infection) (data not shown). Moreover, we failed to detect further inhibition in virus infection when higher concentration (more than $20 \mu \mathrm{g} / \mathrm{mL}$ ) of HBD3 was used in our assay (data not shown). In all the above described experiments, uninfected A549 cells treated with HBDs $(40 \mu \mathrm{g} / \mathrm{mL})$ for eight hours at $37^{\circ} \mathrm{C}$ did not demonstrate cytotoxicity (data not shown). These results suggested that HBD3 confers its antiviral activity by binding to the cell surface and destabilizing viral envelope.

\section{HBD3 inhibits cellular entry of VSV}

We next focused our attention on the ability of HBD3 to restrict cellular entry of VSV, since such activity of defensins has been noted for HIV-1, herpes simplex virus (HSV), influenza and RSV. ${ }^{19-21,31-33}$ We utilized enhanced GFP (eGFP)-expressing pseudotyped lentiviruses (HIV-1 backbone) harboring VSV envelope (G) protein (lentiVSV) to study viral entry. Recombinant lentiviruses pseudotyped with various viral envelope proteins have been previously used to study viral entry. ${ }^{14,34}$ The advantage of using these viruses is that they are nonreplicating and the expression of marker gene (eg, for GFP) serves as a tool to calculate entry efficiency via the envelope protein used for pseudotyping. For our studies, HBD3 and HBD1 (20 $\mu \mathrm{g} / \mathrm{mL}$ )-treated (for three hours) A549 cells were transduced with lenti-VSV. Following five hours adsorption, fresh medium was added to washed cells in the absence of HBDs. At 40 hours post-transduction, expression of eGFP was monitored by fluorescence microscopy. Our results indicate that HBD3 dramatically inhibited cellular entry of VSV as observed by a significant decrease in eGFP expression in cells treated with HBD3 compared to untreated (Figure 3) and HBD1-treated (data not shown) cells.

The inhibition in eGFP expression was not due to inhibition in alternate stages (especially the mode of nuclear targeting and reverse-transcription of the gene in lenti-VSV will be achieved by the mechanism utilized by HIV-1) in viral life-cycle, since a) the major inhibitory mechanism of HBD3 is primarily at the entry stage of HIV-1, ${ }^{19,33} \mathrm{~b}$ ) addition of HBD3 at post-transduction time-frames (2-12 hours post-transduction) does not inhibit eGFP expression (data not shown) and, c) HBD3 was present only during adsorption stage and transduction was performed in the absence of HBD3.

\section{Reduced antiviral activity of IFN- $\alpha$ in HBD3 silenced cells}

Our studies, thus, indicated that IFN- $\alpha$ induces HBD3, which inhibited VSV infection, probably at the stage of viral entry. Based on these observations, we speculated that HBD3 may constitute one of the IFN- $\alpha$ inducible antiviral protein in epithelial cells. In order to verify the role of HBD3 during antiviral function of IFN- $\alpha$, we examined the antiviral efficiency of IFN- $\alpha$ in cells silenced for HBD3 expression. Silencing of HBD3 expression was achieved by transfecting HBD3 specific siRNA. Following 36 hours post-transfection, cells were treated with IFN- $\alpha$ (eight hours treatment) and induction of HBD3 was monitored by qPCR. We noted significant reduction in HBD3 mRNA levels in cells (IFN- $\alpha$ treated) transfected with HBD3 specific siRNA compared to cells
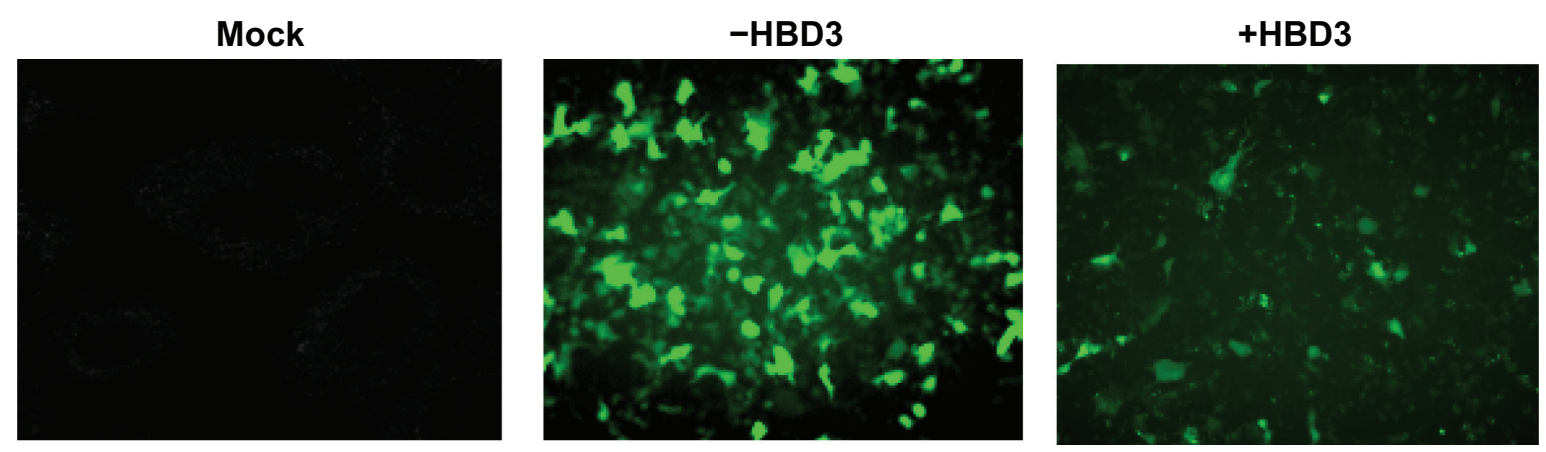

\section{+VSV G Lentivirus}

Figure 3 HBD3 inhibits cellular entry of VSV. Confocal immunofluorescence microscopic analysis of eGFP expression in mock-transduced cells and in cells transduced with VSV G-pseudotyped lentivirus with (+HBD3) or without (-HBD3) pre-treatment with HBD3 for 3 h. HBD3 was absent during lentivirus transduction. 
A

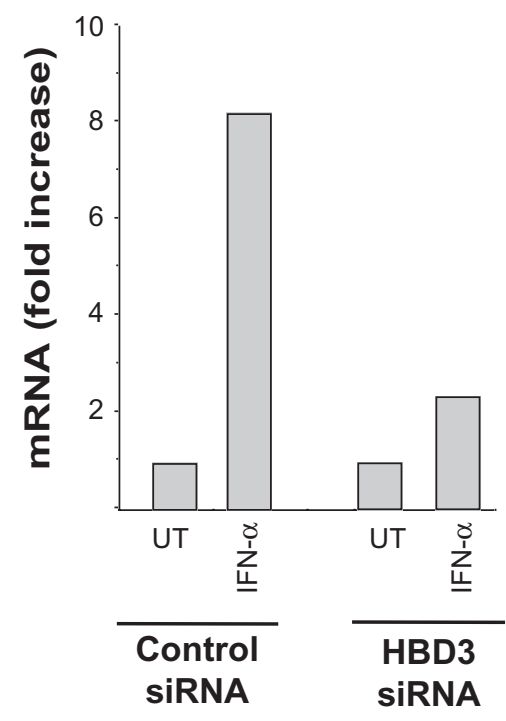

C

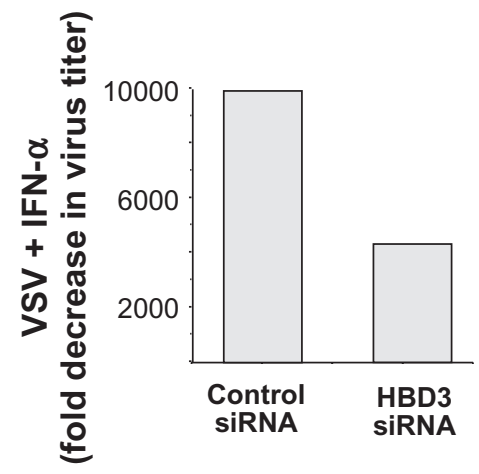

B

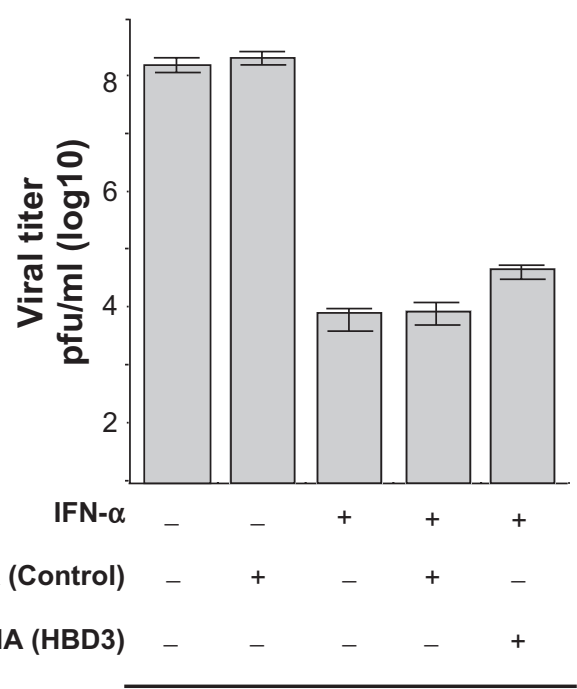

VSV

Figure 4 Role of HBD3 during antiviral function of IFN- $\alpha$. A) qPCR analysis of HBD3 expression in untreated (UT) and IFN- $\alpha$-treated cells transfected with either control siRNA (con siRNA) or HBD3 specific siRNA. B) Cells transfected with either control siRNA or HBD3 specific siRNA were pre-treated with IFN- $\alpha$ (I000 units/ml for 8 h), followed by VSV infection for $36 \mathrm{~h}$. Viral titer was measured by plaque assay analysis and the plaque assay values represent the mean \pm standard deviation for three determinations. C) The plaque assay values from Figure 4B were tabulated to demonstrate the antiviral efficiency of IFN- $\alpha$ in control and HBD3 silenced cells. The values are represented as fold-decrease in viral titer following treatment of control and HBD3 silenced cells with IFN- $\alpha$. D) Western blot analysis of VSV P protein expression in control siRNA and HBD3 siRNA transfected infected cells + /- IFN- $\alpha$ treatment.

(IFN- $\alpha$-treated) expressing the control siRNA (Figure 4A). We next utilized these cells to measure antiviral activity of IFN- $\alpha$. For these studies, untransfected and siRNA transfected cells were either untreated or treated with IFN- $\alpha$ (eight hours treatment). Following IFN- $\alpha$ treatment, cells were infected with VSV in the presence of IFN- $\alpha$. At 36 hours post-infection, plaque assay analysis of medium supernatants obtained from these cells revealed as expected dramatic decline in infectivity (four logs or 10,000 -fold reduction in viral titer) in cells treated with IFN- $\alpha$ compared to untreated cells (Figure 4B). Such decrease in viral titer was also observed in IFN- $\alpha$-treated control siRNA-expressing cells (Figure 4B). However, the antiviral efficiency of IFN- $\alpha$ was substantially reduced in cells expressing HBD3-specific siRNA, since only 4000fold reduction in viral titer was noted in IFN- $\alpha$-treated HBD3-silenced cells (Figure 4B). A comparison of the fold decrease in viral titer (as determined by plaque assay results from Figure 4B) 1 IFN- $\alpha$ treatment of control and HBD3 silenced cells is shown in Figure 4C. Western blot analysis (Figure 4D) of cell lysates with VSV P antibody confirmed the plaque assay results. While complete abolishment of intracellular VSV P protein was observed in IFN- $\alpha$-treated control siRNA-expressing cells, significant levels of $P$ protein was visible in IFN- $\alpha$-treated HBD3 specific siRNA expressing cells. These results, 
thus, indicate that HBD3 plays an important role during antiviral action of IFN- $\alpha$.

\section{Discussion}

Our interest in identifying type-I IFN-induced antiviral factors stemmed from previous observation that apart from PKR, RNaseL, and Mx, additional IFN- $\alpha / \beta$-induced antiviral factors are required to inhibit NNS viruses. ${ }^{10-12}$ Our rationale to focus on HBD3 as a putative IFN-inducible antiviral protein was based on several factors including, a) HBD3 gene harbors STAT-1-binding sequences, ${ }^{25}$ b) HBD3 expression is induced by IFN- $\gamma$ treatment of keratinocytes, ${ }^{35,36} \mathrm{c}$ ) antiviral activity of HBD3 has been demonstrated against HIV-1 and influenza virus, ${ }^{19,20}$ and d) HBD3 is specifically expressed in epithelial cells, ${ }^{17,26-28}$ the cells utilized by us to study virus-host interaction. Our current studies have identified HBD3 as an IFN-inducible antiviral protein. We show induction of HBD3 following IFN- $\alpha$ treatment of human lung epithelial cells (Figure 1). Further more, purified HBD3 significantly inhibited VSV infection by virtue of blocking viral cellular entry antiviral activity against VSV (Figures 2, 3). An important role of HBD3 during IFN- $\alpha$-mediated antiviral response was evident from loss of antiviral efficiency of IFN- $\alpha$ following HBD3 silencing by siRNA (Figure 4).

Defensin family of polypeptides, constituting of alpha, beta and theta defensins are cysteine rich peptides possessing antibacterial and antiviral activity. ${ }^{16-18}$ Human beta-defensins (HBDs) are compact biologically active cationic polypeptides of $40-50$ amino acids (4-7 kDa) with distinctive molecular framework characterized by six cysteine residues paired in three disulfide bridges. The three well-established HBDs belonging to the beta-defensin family of polypeptides are HBD1, HBD2, and HBD3. Apart from the antimicrobial activity of HBDs, we and others have demonstrated potent antiviral function of HBDs against HIV-1, RSV, and influenza virus. ${ }^{19-21}$ Recently, we demonstrated that interaction of HBD2 with RSV envelope results in loss of infectivity as a result of inhibition in cellular entry due to permeabilization/disruption of viral envelope. ${ }^{21}$ The antiviral activity of HBD2 was also noted for VSV (data not shown). Similar antiviral mechanism of HBD2 and HBD3 against HIV-1 has been proposed previously. ${ }^{19}$ Moreover, treatment of cells with HBD2 and HBD3 resulted in inhibition of HIV-1 cellular entry by virtue of downregulation of HIV-1 cellular receptor from the cell surface and/or coating of plasma membrane by HBDs leading to inaccessibility of the HIV-1 cell surface receptor. ${ }^{33}$ Apart from HBDs, alpha and theta defensins also restricted HSV and HIV-1 replication, respectively by inhibiting cellular entry of these viruses. ${ }^{19-21,31-33}$ Nevertheless, these studies have established a potent antiviral activity of defensins against broad spectrum of virus. Our current studies have extended these observations to demonstrate a critical function of HBD3 during innate immune response against virus infection. Our studies suggest that HBD3 may constitute an important IFN- $\alpha$ induced antiviral factor.

The antiviral specificity of HBD3 is borne out by the observation that HBD1 failed to inhibit VSV infection. The inability of HBD1 to restrict virus infection was also noted for HIV-1and RSV. ${ }^{19,21}$ The lack of antiviral activity of HBD1 could be due to its constitutive expression (in contrast to inducible expression of HBD3). In that context, it is possible that although HBD1 will interact with the cell surface and the viral envelope by virtue of its cationic nature, it may fail to mask the cellular receptor of VSV and destabilize the envelope to yield conformationally inactive VSV G protein. A similar phenomenon was observed with another cationic peptide, melittin. It was demonstrated that binding of melittin to VSV envelope results in permeabilization of the envelope and formation of pores. ${ }^{37}$ However, melittin-treated virus retained its infectivity, suggesting that a massive perturbation of envelope is required for compromising infectivity. Such destabilization of the envelope may be achieved by HBD3 and not by HBD1. It is interesting to note that although IFN- $\gamma$ activates STAT1, it failed to induce HBD3 mRNA in lung epithelial cells. Moreover, previously IFN- $\gamma$-mediated induction of HBD3 was noted in keratinocytes. ${ }^{35,36}$ The difference in inducibility of HBD3 could be due to discrete transcriptional complexes formed following cellular treatment with IFN- $\alpha / \beta$ (STAT1/STAT2 hetero-dimer) and IFN- $\gamma$ (STAT1 homo-dimer). It is also possible that although these complexes will include STAT1, other transcriptional factors may be required for efficient expression of HBD3 gene which is lacking in IFN- $\gamma$-treated cells. In addition, IFN- $\alpha$ - and IFN- $\gamma$-mediated HBD3 stimulation could be cell-type dependent (eg, keratinocytes vs lung epithelial cells).

In summary, our current studies have demonstrated that significant amount of antiviral function of IFN- $\alpha$ is attributable to HBD3. Thus, HBD3 represents an IFNinducible antiviral factor that inhibits virus infection by blocking viral cellular entry. Future studies aimed at characterizing the antiviral function of HBD3 in IFN- $\alpha$ treated cells and the mechanism involve in induction of HBD3 in epithelial cells will lead to better understand- 
ing of the innate immune antiviral function of IFN- $\alpha / \beta$ in epithelial cells.

\section{Acknowledgments}

This work was supported by National Institutes of Health grants AI069062 (to S.B), CA129246 (to SB), AI32027 (to AKB) and American Lung Association National Biomedical Research Grant-RG-49629-N (to SB). We greatly appreciate the gift of purified human beta defensins from Dr Miguel E Quinones-Mateu (Cleveland Clinic, Cleveland OH, USA and Diagnostic HYBRIDS, Athens, OH, USA). We would also like to thank Dr. Ratan Maitra (Cleveland Biolabs Inc, Buffalo, NY, USA) for constructing the VSV-G protein pseudotyped lentivirus. The authors report no conflicts of interest in this work.

\section{References}

1. Stark GR, Kerr IM, Williams BRG, Silverman RH, Schreiber RD. How cells respond to interferons. Annu Rev Biochem. 1998;67: 227-264.

2. Vilcek J, Sen GC. Interferons and other cytokines. In: Fields BN, Knipe DM, Howley PM, editors. Fields Virology, 3rd ed. Philadelphia, PA: Lippincott-Raven Publishers; 1996. pp. 375-399.

3. Silverman RH. 2-5A-dependent RNase L: a regulated endoribonuclease in the interferon system. In: D'Alessio G, Riordan JF, editors. Ribonucleases: Structure and function. New York, NY: Academic Press; 1997. pp. 515-551.

4. Clemens MJ, Elia A. The double-stranded RNA-dependent protein kinase PKR: structure and function. J Interferon Cytokine Res. 1997; 17:503-524.

5. Haller O, Kochs G. Interferon-induced Mx proteins: dynamin-like GTPases with antiviral activity. Traffic. 2002;10:710-717.

6. Zhao H, De B, Das T, Banerjee A. Inhibition of human parainfluenza virus-3 replication by interferon and human MxA. Virology. 1996;220:330-338.

7. Staeheli P, Pavlovic J. Inhibition of vesicular stomatitis virus mRNA synthesis by human MxA protein. J Virol. 1991;65: 4498-4501.

8. Stojdl DF, Abraham N, Knowles S, et al. The murine double-stranded RNA-dependent protein kinase PKR is required for resistance to vesicular stomatitis virus. $J$ Virol. 2000;20:9580-9585.

9. Behera AK, Kumar M, Lockey RF, Mohapatra SS. 2-5 Oligoadenylate synthetase plays a critical role in interferon-gamma inhibition of respiratory syncytial virus infection of human epithelial cells. $J$ Biol Chem. 2002;28:25601-25608.

10. Choudhary S, Gao J, Leaman D, De B. Interferon action against human parainfluenza virus type 3 : involvement of a novel antiviral pathway in the inhibition of transcription. J Virol. 2001;75:4823-4831.

11. Zhou A, Paranjape JM, Der SD, Williams BR, Silverman RH. Interferon action in triply deficient mice reveals the existence of alternative antiviral pathways. Virology. 1999;258:435-440.

12. Pattyn E, Van Ostade X, Schauvliege L, et al. Dimerization of the interferon type I receptor IFNaR2-2 is sufficient for induction of interferon effector genes but not for full antiviral activity. $J$ Biol Chem. 1999;274:34838-34845.

13. Dong B, Zhou Q, Zhao J, et al. Phospholipid scramblase 1 potentiates the antiviral activity of interferon. $J$ Virol. 2004;78:8983-8993.

14. Basu M, Maitra RK, Xiang Y, Meng X, Banerjee AK, Bose S. Inhibition of vesicular stomatitis virus infection in epithelial cells by interferon-alpha induced soluble secreted proteins. J Gen Virol. 2006; $87: 2653-2662$.
15. Marcus P, Sekellick MJ. Interferon Action III. The rate of primary transcription of vesicular stomatitis virus is inhibited by interferon action. J Gen Virol. 1978;38:391-408.

16. Dhople V, Krukemeyer A, Ramamoorthy A. The human betadefensin-3, an antibacterial peptide with multiple biological functions. Biochim Biophys Acta. 2006;1758:1499-1512.

17. Harder J, Bartels J, Christophers E, Schroder JM. Isolation and characterization of human beta-defensin-3, a novel human inducible peptide antibiotic. J Biol Chem. 2001;276:5707-5713.

18. Torres A, Kuchel PW. The $\beta$-defensin-fold family of polypeptides. Toxicon. 2004;44:581-588.

19. Quinones-Mateu ME, Lederman MM, Feng Z, et al. Human epithelial $\beta$-defensin 2 and 3 inhibit HIV-1 replication. AIDS. 2003; 17:F39-F48.

20. Leikina E, Delanoe-Ayari H, Melikov K, et al. Carbohydrate-binding molecules inhibit viral fusion and entry by crosslinking membrane glycoproteins. Nat Immunol. 2005;6:995-1001.

21. Kota S, Sabbah A, Chang TH, et al. Role of human beta-defensin-2 during tumor necrosis factor- $\alpha / \mathrm{NF}-\mathrm{\kappa B}$ mediated innate anti-viral response against human respiratory syncytial virus. $J$ Biol Chem. 2008;283:22417-22429.

22. Bose S, Kar N, Maitra R, DiDonato JA, Banerjee AK. Temporal activation of NF-kB regulates an interferon-independent innate antiviral response against cytoplasmic RNA viruses. Proc Natl Acad Sci U S A. 2003;100:10890-10895.

23. Krisanaprakornkit S, Weinberg A, Perez CN, Dale BA. Expression of the peptide antibiotic human beta-defensin 1 in cultured gingival epithelial cells and gingival tissue. Infect Immun. 1998; 66:4222-4228.

24. Krisanaprakornkit S, Kimball JR, Weinberg A, Darveau RP, Bainbridge BW, Dale BA. Inducible expression of human betadefensin 2 by Fusobacterium nucleatum in oral epithelial cells: multiple signaling pathways and role of commensal bacteria in innate immunity and the epithelial barrier. Infect Immun. 2000;68: 2907-2915.

25. Garcia JR, Jaumann F, Schulz S, et al. Identification of a novel, multifunctional beta-defensin (human beta-defesin 3) with specific antimicrobial activity. Its interaction with plasma membranes of Xenopus oocytes and the induction of macrophage chemoattraction. Cell Tissue Res. 2001;2:257-264.

26. Tomita T, Nagase T, Ohga E, Yamaguchi Y, Yoshizumi M, Ouchi Y. Molecular mechanisms underlying human beta-defensin-2 gene expression in a human airway cell line (LC2/ad). Respirology. 2002;7:305-310.

27. Singh PK, Jia HP, Wiles K, et al. Production of beta-defensins by human airway epithelia. Proc Natl Acad Sci U S A. 1998;95: 14961-14966.

28. Duits LA, Nibbering PH, van Strijen E, et al. Rhinovirus increases human beta-defensin-2 and -3 mRNA expression in cultured bronchial epithelial cells. FEMS Immunol Med Microbiol. 2003;38:59-64.

29. Ishimoto $\mathrm{H}$, Mukae $\mathrm{H}$, Date $\mathrm{Y}$, et al. Identification of hBD-3 in respiratory tract and serum: the increase in pneumonia. Eur Respir $J$. 2006;27:253-260.

30. Tao R, Jurevic RJ, Coulton KK, et al. Salivary antimicrobial peptide expression and dental caries experience in children. Antimicrob Agents Chemother. 2005;49:3883-3888.

31. Munk C, Wei G, Yang OO, et al. The theta-defensin, retrocyclin, inhibits HIV-1 entry. AIDS Res Hum Retroviruses. 2003;19:875-881.

32. Sinha S, Cheshenko N, Lehrer RI, Herold BC. NP-1, a rabbit alpha-defensin, prevents the entry and intercellular spread of herpes simplex virus type 2. Antimicrob Agents Chemother. 2003;47: 494-500.

33. Feng Z, Dubyak GR, Lederman MM, Weinberg A. Human beta defensin 3-a novel antagonist of the HIV-1 coreceptor CXCR4. J Immunol. 2006; 177:782-786.

34. Jia HP, Look DC, Shi L, et al. ACE2 receptor expression and severe acute respiratory syndrome coronavirus infection depend on differentiation of human airway epithelia. J Virol. 2005;79:14614-14621. 
35. Sørensen OE, Thapa DR, Rosenthal A, Liu L, Roberts AA, Ganz T. Differential Regulation of \{beta $\}$-Defensin Expression in Human Skin by Microbial Stimuli. J Immunol. 2005;174;4870-4879.

36. Harder J, Meyer-Hoffert U, Wehkamp K, Schwichtenberg L, Schroder J. Differential gene induction of human b-defensins (hBD-1, $-2,-3$, and -4 ) in keratinocytes is inhibited by retinoic acid. J Invest Dermatol. 2004;123:522-529.
37. Witt DJ, Naeve CW, Summers DF. Phosphorylation of vesicular stomatitis virus proteins as a possible contributing factor in virion uncoating. J Gen Virol. 1981;56:383-391.

International Journal of Interferon, Cytokine and Mediator Research

Dovepress

\section{Publish your work in this journal}

The International Journal of Interferon, Cytokine and Mediator Research is an international, peer-reviewed, open-access, online journal. The focus of the journal is to publish original research, reports, editorials, reviews and commentaries on all aspects of interferon, cytokine and mediators of inflammation from laboratory

science to therapeutic indications and clinical studies. The manuscript management system is completely online and includes a very quick and fair peer-review system, which is all easy to use. Visit http://www.dovepress.com/testimonials.php to read real quotes from published authors.

Submit your manuscript here: http://www.dovepress.com/international-journal-of-interferon-cytokine-and-mediator-research-journal 